\title{
FREQUENCY BASED EFFICIENCY EVALUATION - FROM PATTERN RECOGNITION VIA BACKWARDS SIMULATION TO PURPOSEFUL DRIVE DESIGN
}

\author{
Martin Starke ${ }^{1 *}$, Benjamin Beck ${ }^{2}$, Denis Ritz ${ }^{2}$, Frank Will ${ }^{1 *}$, Jürgen Weber ${ }^{2}$ \\ ${ }^{1}$ Institute of Mechatronic Engineering, Endowed Chair of Construction Machines, Technische Universität Dresden, \\ Münchner Platz 3, 01062 Dresden \\ ${ }^{2}$ Institute of Mechatronic Engineering, Chair of Fluid-Mechatronic Systems, Technische Universität Dresden, \\ Helmholtzstraße 7a, 01062 Dresden \\ *Corresponding author: Tel.: +49 351463 39278; E-mail address: martin.starke@tu-dresden.de
}

\begin{abstract}
The efficiency of hydraulic drive systems in mobile machines is influenced by several factors, like the operators' guidance, weather conditions, material respectively loading properties and primarily the working cycle. This leads to varying operation points, which have to be performed by the drive system. Regarding efficiency analysis, the usage of standardized working cycles gained through measurements or synthetically generated is state of the art. Thereby, only a small extract of the real usage profile is taken into account. This contribution deals with process pattern recognition (PPR) and frequency based efficiency evaluation to gain more precise information and conclusion for the drive design of mobile machines. By the example of an $18 \mathrm{t}$ mobile excavator, the recognition system using Hidden - Markov - Models (HMM) and the efficiency evaluation process by means of backwards simulation of measured operation points will be described.
\end{abstract}

Keywords: process pattern recognition, hidden-markov-model, backward simulation, alternative modelling methods, efficiency evaluation

\section{INTRODUCTOIN}

Hydraulic drives have operating point-dependent efficiency characteristics, which result in userspecific primary energy requirements. For this reason, in addition to knowing the operation profile of each construction machine, it is important to obtain information on the efficiency of each task performed (pattern) in order to incorporate this data into the development process or into the current operation. The energy efficiency of mobile machinery must therefore be analysed and evaluated using frequency-based operation point distributions, taking into account the range of applications of the machine. To achieve this goal, two major areas of work were carried out:

1. The model-based efficiency evaluation of the drive system using frequency-based operation point data of parallel-operated axes on a common hydraulic supply.

2. The process pattern recognition for the automated recording of the frequency as well as start and end times of the patterns.

\section{STATE OF THE ART}

\section{Pattern Recognition}

Pattern recognition with its various mathematical methods and approaches is defined in its entirety as machine learning [1]. The different methods of pattern recognition always have to solve the same task, which follows the same sequence. This includes the data preprocessing (e.g. frequency filtering) of a data signal in the so-called analyzer with subsequent transfer to the characteristic space. These characteristics are assigned to classes. The classification then represents the different patterns, which have to be detected [2]. There is a large number of algorithms that must be selected according to the requirements of the recognition task. (e.g. template matching procedure, artificial neural networks, support vector machines (SVM), decision trees or the nearest neighbour classification, hidden markov models (HMM)). These algorithms are already successfully used in areas such as speech, gesture and handwriting recognition [3-6]. In the field of 
mobile machinery Wünsche showed in [7] the recognition of cycles on a wheel loader. With the help of CAN bus signals, a principal component analysis is carried out using a sliding window. Various activities are identified based on the first principal component. The performed investigations show a high sensitivity of the detection rate to the window size used. It remains open whether the main components are suitable as classification features for the identification of complex motion sequences of several loads operated in parallel.

Keller describes in [8] the possibility to detect a loading cycle of a mini excavator. Due to the simplicity of the algorithm, the method of the decision tree is used. 16 loading cycles are carried out on a test machine and the existing measured variables are recorded. The decision tree algorithm is then trained with this data, which represents the assignment of the activity label. The sub-processes idling, driving, unloading, slewing and digging are labelled. In the subsequent recognition phase, he examines the accuracy of the calculated sequences with $20 \%$ of the recorded data and the influence of the available signals on the machine. In the result, the joystick signals are identified as the most influential signals.

In [9] the suitability of the template matching procedure and HMM to define customer usage profiles are shown. Thereby HMM show the best results for the recognition of sequences of various lengths. This and the other listed examples show only the recognition of individual working cycles. Relevant points such as determining the cycle duration, the detection of different cycles, the learning of unknown processes and further the evaluation of the detected processes are not considered yet. This contribution deals with these issues.

\section{Efficiency evaluation}

Due to the great heterogeneity of mobile machinery, it is very difficult to define specific methods for evaluating energy efficiency. In addition, the machine operators have a major influence on the energy consumption of the machine (design, selected machine size, age of the machine) [10]. Today, however, there are several methods and guidelines for evaluating fuel consumption for different types of mobile machinery. Three approaches can be distinguished:

1. direct fuel measurements [11-14]

2. subsystem tests [15]

3. model-based system analysis [10, 16-23]

While in one measurements are possible in real life and based on defined cycles, the procedures in two and three are limited to reference cycles.

Mieth shows that it seems most reasonable to use a small, representative number from the set of all measured cycles on the basis of the density distribution function for the efficiency evaluation. In this example, a very good agreement in the load distribution on the stick cylinder could be obtained by using only 10 instead of all 92 measured cycles [10]. This underlines the necessity to use a statistically proven set of operation points for the efficiency evaluation. With regard to the traction drive of a mobile machine, a corresponding methodology has been developed in [24, 25]. Jähne sets up simulation models for different transmission structures and derives efficiency maps by varying the model input variables $\left(v_{i}, F_{i}\right)$. These maps can easily be calculated with the frequency data of the operation points in order to determine a system input power. In general, this methodology can be applied to all systems with only one active load. As soon as several consumers are operating simultaneously, the dimension of the efficiency maps increases and with it the simulation effort to generate them. For the calculation of the system input power for given operation points of consumers operated in parallel other calculation methods have to be developed, which are part of this paper. The term "efficiency model" will be introduced to describe the resulting models.

\section{VALIDATION OBJECT}

The validation of the efficiency assessment methodology, including process pattern recognition, was carried out on an A918 mobile excavator from Liebherr. The machine is equipped with a LUDV valve system and a hydraulic-mechanical load-sensing concept for adjusting the pump. Measurement series with 16 cycles for digging with the corresponding flux and potential values at the main loads boom, stick, bucket and swing gear were used. These are shown in extracts in Figure 1. 


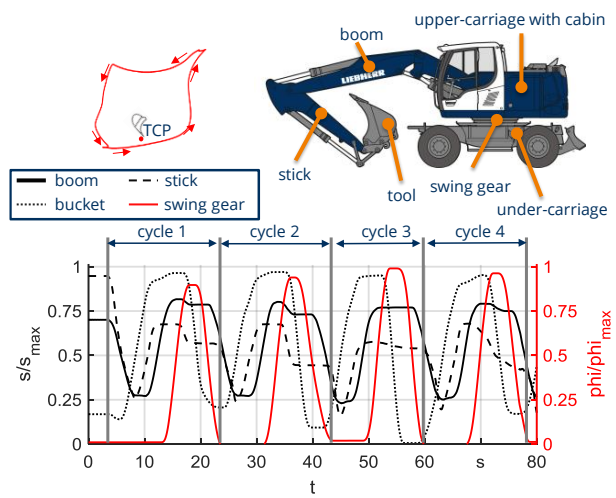

Figure 1: A918 object of examination - digging cycle

This machine and its provided data is used for the development and validation of the shown system in this contribution.

\section{PROCESS PATTERN RECOGNITION}

The Hidden Markov Models (HMM) used in this publication are particularly well suited for an application in the area of mobile machinery, as they can also cope with widely varying cycle times and, in addition, an increase in detection quality can be achieved through adaptation during operation [9].

HMM are stochastic calculation models, which have the characteristic to assign states to observable parameters (sensor signals). For this purpose, the process pattern (set of similar working cycles) to be determined can be allocated to the order of a time sequence of states, which can assume any number $n$.

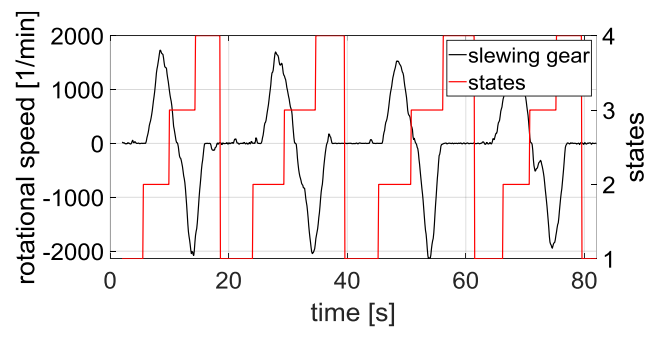

Figure 2: sensor signal and calculated state sequence

Figure 2 shows this context with the calculation of $n=4$ states to the time sequence of four digging cycles represented in the rotational speed of the slewing gear. If the HMM calculates the sequence of states, a subsequent evaluation algorithm can determine the pattern out of the order of state sequence.
Before this recognition can be performed, the HMM hast to be trained. Therefore, the sequence of states to be calculated must be assigned to the training data, which must be the same sensor signal as in the detection phase. In the training phase the HMM learns the allocation of the predefined state sequence and the sensor signal. A problem with this procedure is that not every sensor signal is suitable for a high calculation quality of the state sequence. It depends on the spread of the measured values between two different states, which has to be significantly greater than the spread yielded by the sensor within a state. This behavior is varying for the different working cycles. It leads to the first problem that unknown patterns, which the HMM does not know, could not be used for a training without an elaborate statement of suitability. Due to this a new method, called quadrant method $(\mathrm{QM})$, of data preprocessing was introduced in [26]. The approach was chosen due to the requirement of the independence of certain sensor signals. The basic idea is the regard of every single load and its working point in the speed-force- respectively speed-torque-diagram (flux and potential values). Not the absolute values are examined, but only the appearance in the quadrant of the particular diagram of the load in every time step is observed. By computing the combination of all loads in every time step a sequence is achieved, which is the substructure both of the training and of the application of the HMM. In Figure 3, the combination sequence with 3 loads (boom, stick, bucket cylinder) of the 16 cycles of the A918 is presented.

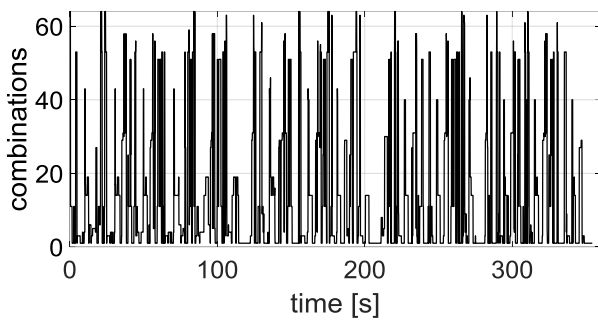

Figure 3: combination sequence of 16 digging cycles

The next step is the state assignment to this sequence before HMM training can be done. As well as the so far described functions, this is realized in Matlab ${ }^{\circledR}$ by an algorithm in the following named autostate function. The functionality aims to the possibility of an 
automatic state assignment for new patterns, which are unknown. Especially for an online implementation this is necessary. The approach that is chosen is known from handwriting recognition. Here, stair-like state progressions are used for observation sequences of different lengths, which are represented by different symbols, letters and operators. The idea is now to assign a staircase to a process pattern and use it for the training of the HMM. The number of states is freely selectable and influences the configuration of the HMM regarding fluctuating detection rates.

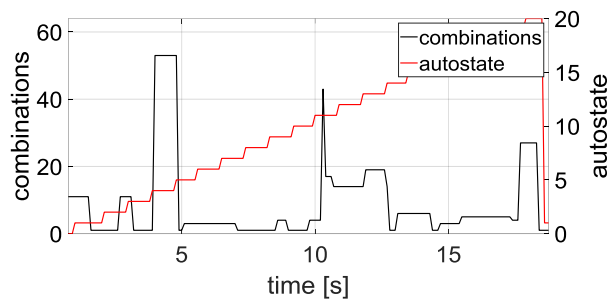

Figure 4: autostate assignment for one cycle

\section{Counting of patterns}

The counting of the patterns consists of the determination of start and end times and the calculated duration. This also results in the number of occurring patterns. For this purpose, the state sequence with 20 states shown in Figure 4 is used (one cycle). The advantage is the possibility of counting the sequences calculated by the HMM afterwards in the recognition phase, which in the best case can be computed exactly as a staircase sequence. Figure $\mathbf{5}$ shows this approach exemplarily for four states. The counting event is triggered when all states have been passed through one after the other and the stack is filled. This method makes it very easy to determine the times and thus the durations of each pattern, since both the start time with the beginning of the stack filling and the end time with the filled stack are available.

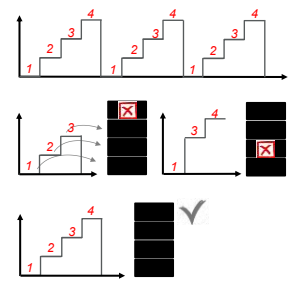

Figure 5: principle of the memory stack

\section{Operation point storage}

Since QM already uses the flux and potential values of each load, the operation points are known at the time the pattern occurs. However, due to the storage requirements, the operation point storage has a high significance. For the transfer of the time-based continuous data stream into the frequency distributions required for the efficiency evaluation, the storage of the operation points using multidimensional classification with dynamically growing list is applied. With this method only operation points that actually occur are stored for every pattern. This must be done during online recognition parallel to the actual recognition. The model must detect the beginning of a pattern and fill the dynamic list during recognition. After the recognition and thus the assignment to a pattern, the newly occurring operation point combinations extend the list of this pattern.

\section{Evaluation}

The model is designed so that it can be used both online and offline as a data evaluation tool. The current state of development is the possibility of recognizing an automatically trained pattern and the saving of the operation points with their start and end times for the efficiency evaluation. In a reduced offline version, the recognition of different trained and unknown patterns is possible. However, in this model the start and end times can only be determined with insufficient accuracy. For this reason, this publication uses the online model that is implemented in Simulink. Figure 6 shows the recognition result of 16 digging cycles of the Liebherr A918 with 64 trained states. The recognition rate is $93 \%$ because 15 of the 16 cycles were detected by the HMM. The red line is the calculated state sequence, were the stairway of the trained sequence is identifiable. The last cycle is not detected, because no following cycle let the HMM calculate a state which indicates the end of this sequence and the start of the new one. 


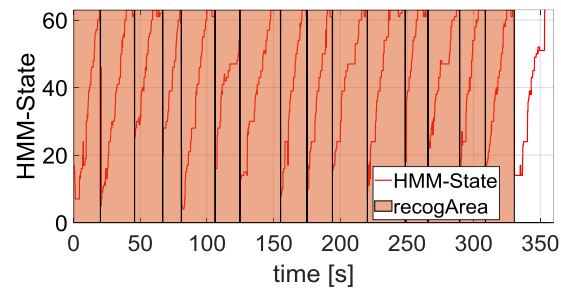

Figure 6: detection result of Liebherr A918 data

The interesting thing is the result shown in Figure 7, where the detection result of the excavator CASE WX 185 data is presented. This data contains 8 cycles of digging and 8 cycles of levelling. The HMM which was used for the detection is the same like in the first example. Due to the usage of the QM method, which uses the separate loads of the machine at least 5 of the 8 included cycles are detected $(2,3,5,6,7)$. Cycle $1 \& 4$ are detected, but with wrong cycles times. The last cycle detected (8) is a wrong detection. The levelling cycles weren't determined.

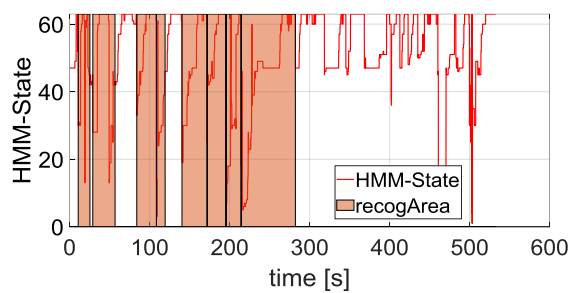

Figure 7: detection result of Case WX 185 data

The stairway is not as well calculated as in Figure 6 but nevertheless the individual cycles can be identified and detected by the HMM. This is caused by the completely different general conditions, which are a different machine, another operator, a varying time and a differing load.

This model was implemented on a control unit (STW ESX-3XM) and is used in a hardware-inthe-loop test bench and on the excavator. As the excavator A918 was not available for validation purposes, the excavator CASE WX 185 and a corresponding real-time capable model were used. Since QM can be used, as shown before, across all machines, the application can also be carried out on multiple machines. With that online application the shown detection results can be achieved in real time and the operation points and the duration of every single cycle are saved in matrix and are available for the efficiency evaluation described in the following chapter.

\section{EFFICIENCY EVALUATION}

An important prerequisite for efficiency analysis is the choice of the system boundary. For the investigations in this publication the mechanical power of the actuators (cylinders, motors) represents the one system boundary. A measurement of cylinder or motor pressures can be easily realized in practice. These should be used to calculate the occurring cylinder force / motor torque. Sensors in the course of automation on the machines partly already record the speeds and revolutions of the output drives. If this is technically more difficult, as for example with bucket cylinders on excavators, it is assumed that estimated values from the measured joystick deflection are available. For a practicable efficiency model, the other system boundary should be designed flexibly depending on the available data for modelling or measurement and should be located between fuel consumption at the engine, mechanical shaft power of the engine and hydraulic power of the pump(s). In principle, it is also conceivable to equip the machine with maximum sensor technology and to make simple monitoring possible over the period of use. However, this can only provide information depending on the currently installed system. The following is possible by measuring the mechanical output power and converting it via a model of the drive system (efficiency model) to the required input power and other intermediate variables:

1. calculation of the necessary input power depending on the selected system boundary

2. analysis of the loss points in the system

3. assistance in the engineering process to find the energetically optimal drive structure for certain tasks. 
The special feature compared to the classical simulation is the backward calculation of the state variables in the system based on the measured operation points. Whereas in the classical system simulation, for example, the motor speed, pump swivel angle and deflection of the valve spools are pre-set, whereupon the speeds of the axes are adjusted according to the acting loads, these variables must now be calculated based on the pre-set forces and speeds. The principle of the efficiency model is displayed in Figure 9.

For practicability, the efficiency model should obtain the static loss behaviour of the system, be easily parameterisable and calculate the solution within seconds.

\subsection{System description}

The load sensing system with secondary pressure compensators is a widespread hydraulic system for mobile excavators on the European market. The test object mobile excavator used here also contains this drive system. In detail, it contains of a hydraulic-mechanically controlled load-sensing pump connected to a diesel engine, a LUDV valve block, pipe breakage safety valves and hydraulic-mechanically Joysticks. According to the $90^{\circ}$-digging cycle, the main consumers boom, stick, bucket and slew drive, which are all valvecontrolled on a single pump are taken into account. Because the backwards calculation of the hydraulic pressure at the pump is the biggest problem, this is the focus in the following. A simplified hydraulic circuit diagram of an exemplary chosen two consumer LUDV-system is displayed in Figure 8.

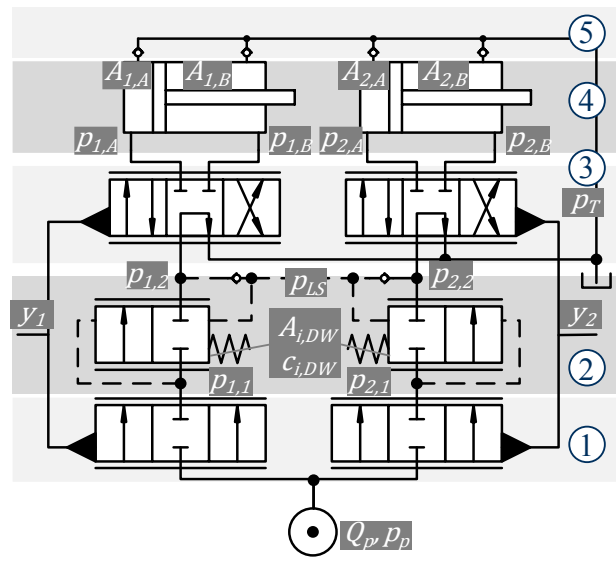

Figure 8: simplified hydraulic circuit diagram of a LUDV system

It can be divided into five sections with the following stationary element equations:

1. Metering orifices (MO)

$Q_{i, M O}=K_{i, M O} \cdot A_{i, M O}\left(y_{i}\right) \cdot \sqrt{p_{p}-p_{i, 1}}$

2. Individual pressure compensators (IPC)

$Q_{i, I P C}=K_{I P C} \cdot A_{I P C}\left(y_{i, I P C}\right) \cdot \sqrt{p_{i, 1}-p_{i, 2}}$

$y_{i, I P C}=\frac{A_{i, I P C}}{c_{i, I P C}} \cdot\left(p_{i, 1}-\max \left(p_{i, 2}\right)\right)$

3. Direction edges (DE)

$$
\begin{aligned}
& Q_{i, D E_{-} u p}=K_{D E_{-} u p} \cdot A_{D E_{-} u p}\left(y_{i}\right) \\
& \cdot \sqrt{p_{i, 2}-p_{i, A / B}} \\
& Q_{i, D E_{-} \text {down }}=K_{D E_{-} \text {down }} \cdot A_{D E_{-} \text {down }}\left(y_{i}\right) \text {. } \\
& \sqrt{p_{i, 2}-p_{i, A / B}}
\end{aligned}
$$

4. Actuators indexed with ascending alphanumeric numbers

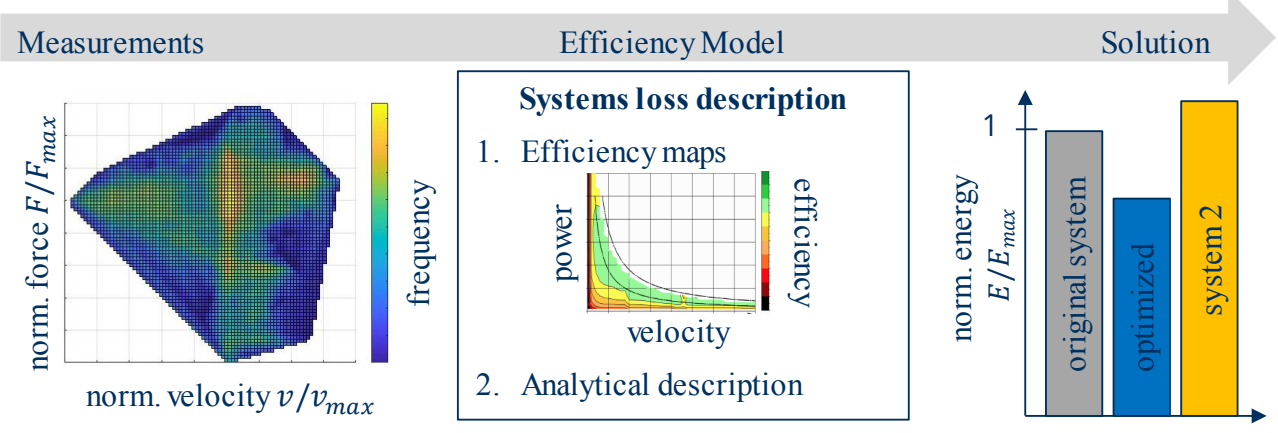

Figure 9: concept of efficiency modelling 
$F_{i, z y l}=p_{i, A} \cdot A_{i, A}-p_{i, B} \cdot A_{i, B}$

$v_{i}=\frac{Q_{i, A}}{A_{i, A}}=\frac{Q_{i, B}}{A_{i, B}}$

5. Suction valves (SV)

$Q_{i, S V}=K_{S V} \cdot A_{S V} \cdot \sqrt{p_{T}-p_{i, A / B}}$

Besides this, the following balance equations are valid:

6.

$F_{i}=F_{i, c y l}$

$Q_{i, A / B}=Q_{i, D E \_u p}+Q_{S V}$

$Q_{i, B / A}=Q_{i, D E_{-} \text {down }}+Q_{S V}$

$Q_{i, D E \_u p}=Q_{i, I P C}$

$Q_{i, I P C}=Q_{i, M O}$

$Q_{p}=\sum Q_{i, M O}$

$p_{p}=\max \left(p_{i, 2}\right)+\Delta p_{L S}$

Thereby, the cylinder chamber pressure $p_{i, A / B}$, the intermediate pressures $p_{i, 1 / 2}$, the valve stroke $y_{i}$, the IPC stroke $y_{i, I P C}$ and the pump pressure $p_{p}$ are unknwokn.

It becomes obvious that the system of equations is solvable in principle, since determinacy exists (seven equations and seven unknown variables). Due to the discontinuities (opening of suction valves, maximum load pressure in eq. (3)), the non-linearity's (root function in flow equations and $A(y)$ characteristic of the control edges), a direct inversion of the input and output variables is impossible.

To overcome this issue, three different variants of efficiency models were developed, named as:

1. Serial Iteration

2. Parallel Iteration

3. Blackbox Model

The methods one and two belong to the category of light-grey box models, as they are based on the static system equations and contain characteristic curves of the valves. Both methods use the equations described above. The serial iteration uses the bisection method for iterative solutions. With the "parallel iteration" model, the approach is to use existing "classical forward models" and vary the potential quantities until all balance equations are approximately zero. For this purpose, a multidimensional Newton iteration method is applied. The disadvantage of this method is that the solution of the Newton method depends strongly on the starting value of the iteration. This is contrasted by the method of black box modelling. The idea here is to train the relationship between the operating points at the consumers and at the pump based on measured data or an already existing complex system simulation models. This model can then be used to quickly simulate further operation points. However, physical parameterisation is not necessary. The three methods were implemented in Matlab ${ }$ and then compared with the measured operation points of the pump.

In [27] it could be shown that the black box model requires the least computing time and achieves the highest accuracy. However, the accuracy decreases strongly with measurement data deviating strongly from the training data set and an interpretability due to missing physical correlations is not given. When comparing the two iterative methods with each other, serial iteration is preferable because of its better accuracy and higher speed. However, parallel iteration is much easier to model and thus to apply, as it is possible to derive the efficiency model from existing classical models. However, the computing times are unacceptably high.

\section{Detailed description of serial iteration model}

The diagram in Figure $\mathbf{1 0}$ shows the basic procedure of the developed program. In principle, this is divided into two calculation phases. In the first part the pump pressure is calculated. To determine the valve stroke, it is assumed that the set LS pressure, which drops across the pressure compensator and the metering orifice, is constant. The volume flow required for the movement, which must be conveyed by the pump, results from the product of the cylinder area and cylinder speed. From the pressure and the volume flow it is possible to determine the valve stroke. Since the pressure compensator and the main valve are not mechanically coupled, the pressure compensator must be balanced hydraulically as well as mechanically. With the so determined valve stroke it is now possible to calculate all other variables backwards from the tank and to get a statement about the required pump pressure for each consumer. The highest determined pump pressure represents the pump pressure that is set. 


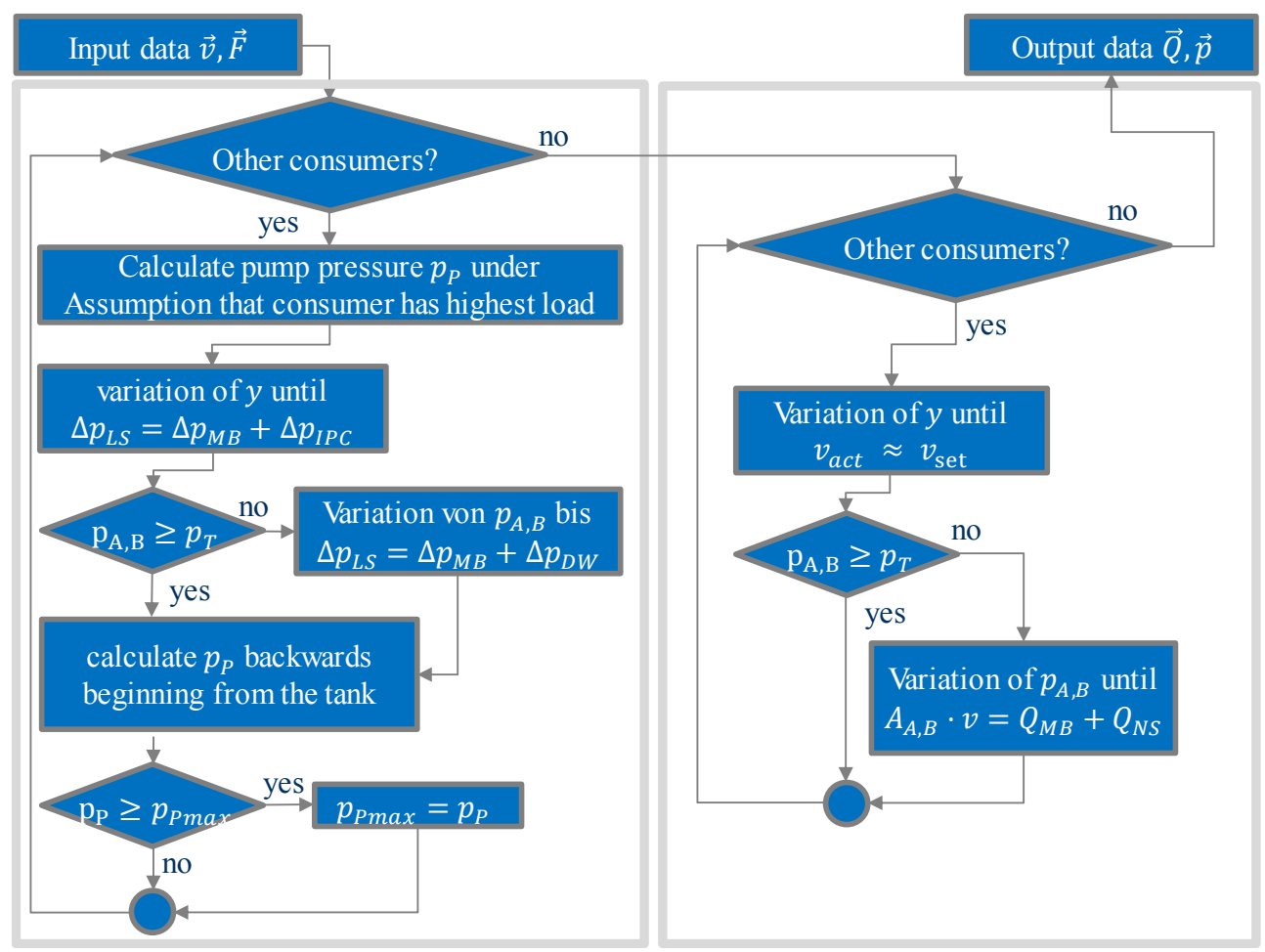

Figure 10: schematic of the developed program for the "serial iteration" efficiency model

$$
v_{c y l}=\frac{A_{P} \cdot p_{p}-A_{T} \cdot p_{T}-F_{c y l}}{A_{P}{ }^{3} \cdot\left(\frac{1}{K_{M O}{ }^{2} \cdot y^{2}}+\frac{1}{K_{D E_{-} u p}{ }^{2} \cdot y^{2}}+\frac{1}{K_{I P C}{ }^{2} \cdot y_{I P C}{ }^{2}}\right)+{A_{T}}^{3} \cdot\left(\frac{1}{{K_{D E} d o w n}^{2} \cdot y^{2}}\right)}
$$

In the second part of the program, the volume flows, pressures and valve strokes of the lowerload consumers are calculated. Here, the valve stroke is varied until the speed of the cylinder at a given pump pressure almost corresponds to the measured value. For this purpose the static control law is used according to the following equation:

\subsection{Special Modelling aspects}

\section{Control edge geometry}

The mapping of the opening cross section as a function of the valve stroke $A(y)$ plays a central role, especially in the field of mobile machinery, since the valves are designed for the operatorguided process and thus include both a fine control range and a range of high speeds in combination with a positive overlap. In addition, the control edges have different characteristics to intercept conditions such as pulling loads. For the modelling it has turned out to be advantageous to ignore the positive overlap range at first, i.e. not to calculate system states. This is realized by simple IF-THEN-ELSE queries. Furthermore, it is advantageous to carry out a normalization from zero to one. The volume flow amplification $K_{i}$ of the individual control edges contains, on the one hand, the flow coefficient $\alpha$, which is an empirical value to take into account all losses such as fluid friction or turbulence. This factor depends mainly on the Reynolds number and the geometry of the control edges. Realistic values for valves are in the range between 0.6 and 0.8 , whereby a good approximate value of $0.7 \mathrm{can}$ be assumed. On the other hand $K_{i}$ contains the density, which is assumed to be $\rho=880 \mathrm{~kg} / \mathrm{m} 3$. 


\section{Input pressure compensator}

This is relatively simply implemented by an IFTHEN-ELSE query. A fixed volume flow rate value is calculated at the set LS pressure differential if no consumers are moved.

\section{Secondary pressure limitation}

The secondary pressure relief valves are coupled to the control signal of the valves. They limit the load pressure $p_{L}$ when the valve is closed to a fixed set value $p_{\text {closed. }}$. When the valve is fully open, the maximum load pressure $p_{\max }$ is set. Mathematically, the relationship can be described according to the following equation:

$$
p_{L}=\left(p_{\max }-p_{\text {closed }}\right) \cdot \frac{y}{y_{\max }}+p_{\text {closed }}
$$

\section{Regeneration position in the main valves}

Some of the valve systems installed today already feature section-internal regeneration between the cylinder chambers due to improved energy efficiency of the machine. This is particularly the case with the boom and stick. However, this poses a major challenge for backward calculation with the efficiency model, as there is no longer a direct correlation between cylinder speed and volume flow over the directional edges. The system is therefore under-determined and cannot be calculated. There are two possibilities to solve this problem:

1. assumption of a fixed volume flow distribution

2. measuring another system parameter and feeding it back into the efficiency model

Solution 1 is to be preferred, since measuring another variable within the system does not correspond to the philosophy of the efficiency model as described above. However, in the case under investigation this could only be applied to the boom cylinder. For the stick cylinder, the measurement of the pressure in the node of the volume flow distribution had to be resorted to.

\subsection{Results on demonstrator}

The results of the efficiency model for the test object are shown in Figure 11. The accuracy of $2 \%$ of the overall normalized energy consumption of the duty cycle shows that the efficiency model can be used for further investigations. The computing time of $182 \mathrm{~s}$ for 35.761 operation points ( $=357 \mathrm{~s}$ duration) is acceptable and meets the requirement "within a few seconds". A transferability to other systems, especially open-center systems with many volume flow distributions, seems impracticable from the authors' point of view. For this purpose, other methods such as real-time capable, classical
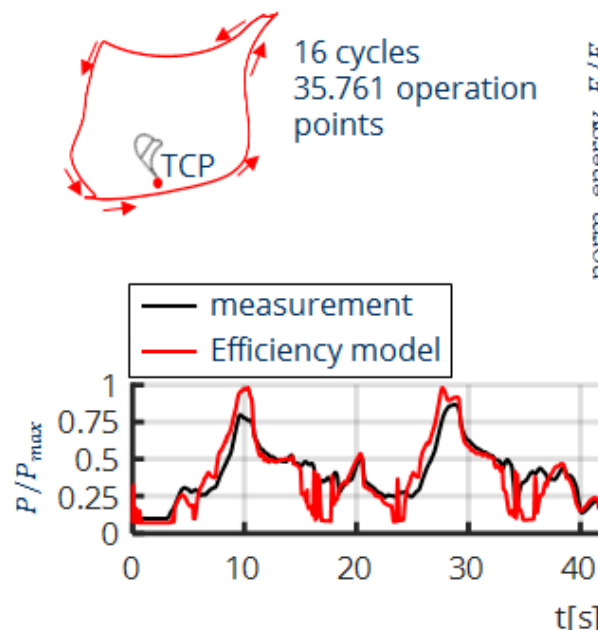

$\mathrm{t}[\mathrm{s}]$

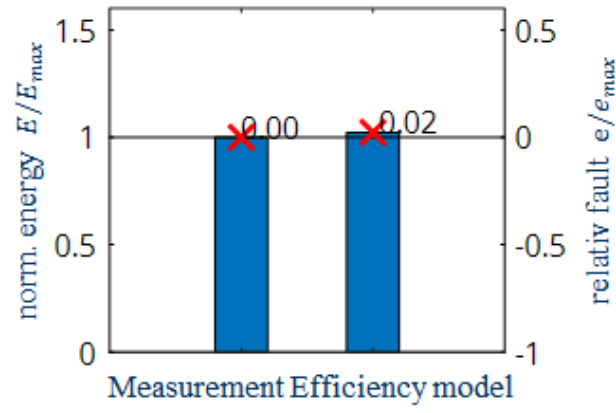

Measurement Efficiency model

Figure 11: Results of "serial Iteration" efficiency model for typical duty cycles of mobile excavator 
forward models or direct energy monitoring with a few model-based components are proposed.

\section{CONCLUSION AND OUTLOOK}

This paper shows a new method that allows the analysis and evaluation of energy efficiency of mobile machinery by means of frequency-based operation point distributions. First, the occurring duty cycles and the frequency of their occurrence are detected by the process pattern recognition (PPR). The duration of the cycles and the operation points contained therein are stored. These are subsequently used to calculate backwards the input power of the drive system through the efficiency model. The two subsystems PPR and efficiency evaluation are combined in one overall system, which makes it possible to achieve a better understanding of the use of machines and the resulting loads on the drive system with minimal effort. It can be assumed that the automated collection and processing of the application data is a continuous process which will not be limited to a single machine development project. The creation of a statistically secured database with a corresponding scope thus serves to build up the basic knowledge for the conception and design of future machine generations or their components and subsystems.

\section{ACKNOWLEDGEMENT}

The project Ref. No. AiF 19613 BR/1 was financed and supervised by the Research Association Mechanical Engineering (FKM). In the scope of the Programme to promote Industrial Collective Research it was funded by the German Federation of Industrial Research Associations (AiF) with means of the Federal Ministry of Economic Affairs and Energy (BMWi) on the basis of a decision by the German Bundestag.

\section{REFERENCES}

1. Aunkofer B Machine Learning vs Deep Learning - Wo liegt der Unterschied? - Data Science Blog. https://data-scienceblog.com/blog/2018/05/14/machine-learningvs-deep-learning-wo-liegt-der-unterschied/. Accessed 20 Dec 2019
2. Hoffmann R (1998) Signalanalyse und erkennung: Eine Einführung für Informationstechniker

3. Dahl G, Yu D, Deng L, Acero A (2011) Contextdependent pre-trained deep neural networks for large-vocabulary speech recognition. IEEE Transactions on audio, speech, and language processing 20:30-42

4. Gales M, Young S (2008) The application of hidden Markov models in speech recognition. Foundations and Trends ${ }^{\circledR}$ in Signal Processing 1:195-304

5. Lizhong G Appearance-based hand gesture recognition and study on human-robot interaction $[\mathrm{D}] . \mathrm{PhD}$ Thesis, Doctoral Dissertation

6. Yun L, Lifeng Z, Shujun Z (2012) A hand gesture recognition method based on multifeature fusion and template matching. Procedia Engineering 29:1678-1684

7. Wuensche M, Mostaghim S, Schmeck H, et al (2010) Organic computing in off-highway machines. In: Proceedings of the second international workshop on Self-organizing architectures. ACM, pp 51-58

8. Keller NJ, Ivantysynova M, Vacca A, et al (2019) Classification of machine functions: a hydraulic excavator case study. In: Proceedings. Tampere, pp 410-422

9. Mieth S, Weber J (2016) Methode zur Online Prozessmustererkennung für die Ermittlung von Kundenkollektiven an mobilen Arbeitsmaschinen. TU Dresden Professur für Baumaschinen, Dresden

10. Kunze G, Mieth S, Voigt S (2011) BEDIENEREINFLUSS AUF LEISTUNGSZYKLEN MOBILER ARBEITSMASCHINEN. ATZoffhighway 4:70-79

11. Helmus M, Fecke M (2015) Standardisierung definierter Lastzyklen und Messmethoden für die Energieverbrauchsermittlung von Baumaschinen. Bergische Universität Wuppertal, Interdisziplinäres Zentrum III

12. Liebherr Wheel Loaders L550 - L586

13. Pfab H, Altenberger F, Gappmaier R (2017) Flottenmessungen zur Optimierung des Maschineneinsatzes. KIT Scientific Publishing, Karlsruhe, p 19

14. Verein deutscher Ingenieure (2012) VDI 2198 Type sheets for industrial trucks

15. Berlenz S (2015) Potentialanalyse bedarfsgerecht betriebener Nebenaggregate an 
einem Off-Highway-Motor. Logos Verlag, Berlin

16. Deiters H (2009) Standardisierung von Lastzyklen zur Beurteilung der Effizienz mobiler Arbeitsmaschinen. Technische Universität Carolo-Wilhelmina zu Braunschweig

17. Filla R (2005) Operator and machine models for dynamic simulation of construction machinery. Linköpings universitet

18. Finzel R (2011) Elektrohydraulische Steuerungssysteme für mobile Arbeitsmaschinen. TU Dresden

19. Fleczoreck $T$ (2013) Effizienzbewertung von Antrieben mobiler Arbeitsmaschinen am Beispiel eines Mähdreschers. Shaker, Aachen

20. Holländer C (1998) Untersuchungen zur Beurteilung und Optimierung von Baggerhydrauliksystemen. VDI-Verl, Düsseldorf

21. Inderelst M (2013) Efficiency improvements in mobile hydraulic systems. Shaker

22. Sitte A, Uhlmann J, Weber J, et al (2017) MODEL BASED EFFICIENCY ANYLYSIS OF MOBILE HYDRAULIC MACHINERY On The Example of Mate rial Handling Machines. JFPS, Fukuoka, p 10

23. Sturm C (2015) Bewertung der Energieeffizienz von Antriebssystemen mobiler Arbeitsmaschinen am Beispiel Bagger. KIT Scientific Publ, Karlsruhe, Baden

24. Jähne H (2009) Simulation of Drive Systems for Mobile Machines. Dresden

25. Jähne H (2013) Struktursystematik und Effizienzpotentiale hydraulischer Fahrantriebe unter Einbeziehung der Applikation. Shaker

26. Starke M (2019) Automatic Process Pattern Recognition for mobile machinery. In: Proceedings. Tampere, pp 450-461

27. Beck B, Koch O, Ritz D, et al (2018) Modellbasierte Entwicklung mobiler Maschinensysteme. In: 7. Fachtagung Baumaschinentechnik 2018. Dresden, pp 185200 